\title{
Le cardinal de Fleury ou comment devient-on le précepteur de Louis XV?
}

How did Cardinal de Fleury become preceptor of Louis XV?

Kardinal Fleury, oder wie wird man Präzeptor von Ludwig XV?

¿El cardenal de Fleury o cómo una persona llega a ser el preceptor de Luis XV?

Peter R. Campbell

\section{(C) OpenEdition}

1 Journals

\section{Édition électronique}

URL : https://journals.openedition.org/histoire-education/2415

DOI : 10.4000/histoire-education.2415

ISSN : 2102-5452

Éditeur

ENS Éditions

\section{Édition imprimée}

Date de publication : 1 octobre 2011

Pagination : 49-72

ISBN : 978-2-84788-360-2

ISSN : 0221-6280

Référence électronique

Peter R. Campbell, «Le cardinal de Fleury ou comment devient-on le précepteur de Louis XV ? », Histoire de l'éducation [En ligne], 132 | 2011, mis en ligne le 01 octobre 2013, consulté le 20 mai 2021. URL : http://journals.openedition.org/histoire-education/2415; DOI : https://doi.org/10.4000/histoireeducation. 2415 


\title{
Le cardinal de Fleury ou comment devient-on le précepteur de Louis XV?
}

\author{
Peter R. CAMPBELL
}

Cet article, qui porte sur la manière dont André-Hercule de Fleury devint précepteur en charge de l'éducation du futur Louis XV, examine les enjeux politiques liés à cette fonction. Mon intention est moins de traiter la pratique pédagogique de Fleury précepteur ${ }^{1}$, que de suivre la route empruntée par Fleury pour accéder à ses hautes fonctions et d'expliquer comment cet habile négociateur fut en mesure d'exploiter sa position d'éducateur pour atteindre le sommet du pouvoir politique ${ }^{2}$. En effet, la place tenue par Fleury dans l'éducation de Louis XV fut certainement décisive dans son ascension vers le pouvoir de 1720 à 1726. Comme favori royal, il acquit une position dans le gouvernement de la France analogue à celle occupée par les cardinaux Richelieu, Mazarin et Dubois. Ses origines étaient pourtant plus modestes que celles des deux grands ministres et il dut attendre plus longtemps pour accéder au pouvoir - si, en vérité, c'était là son intention, car rien n’indique qu'il était aussi " motivé " que ses prédécesseurs. Il serait totalement erroné de prétendre que Fleury se servit délibérément et dès le début de ses fonctions de précepteur pour conquérir le pouvoir politique. Cela fut avancé bien plus tard par des commentateurs qui ont réinterprété les événements à la lumière de sa promotion finale en 1726. La

1 Pour une bibliographie générale sur l'éducation de Louis XV, on se reportera à l'introduction du présent numéro par Diego Venturino.

2 Peter R. Campbell, The conduct of politics in France in the time of the cardinal de Fleury, thèse de doctorat, université de Londres, $1985,1^{\text {re }}$ partie. 
mort du Régent, le choix de Bourbon comme ministre en 1723, les changements entre clans à la Cour, étaient tous imprévisibles en 1717, l'année où le jeune roi passa entre les mains de son précepteur. Fleury était certainement ambitieux, mais au-delà des accusations de duplicité dont il a été l'objet, c'était surtout un clerc habile et compétent, maîtrisant les codes sociaux de la Cour et comprenant assez la politique pour garder profil bas quand cela était nécessaire, comme plus tard pour saisir les opportunités qui se présentaient. Sa meilleure tactique fut de faire son travail de précepteur de son mieux, sans soulever de polémique, et de développer des liens étroits avec son élève. C'était d'autant plus facile que l'enfant royal était en quête d'affection et d'encadrement. Une telle ligne de conduite lui assura une place éminente à la Cour, mais même Fleury pouvait difficilement imaginer qu'il aurait jamais la chance de gouverner la France au nom de son ancien élève.

On étudiera successivement ici les origines et les débuts de la carrière de Fleury, comment il est devenu précepteur et comment il a utilisé cet emploi pour devenir ministre, ce qui nous permettra de réviser quelques jugements admis sur ce personnage.

\section{I - L'ascension de Fleury : un notable languedocien devenu courtisan}

La trajectoire sociale d'André-Hercule de Fleury heurte les représentations dominantes de son temps, dans la mesure où il est un "outsider ". Issu d'une famille modeste du Languedoc, dont les origines ne remontent guère avant 1630, il a dû gravir de nombreux échelons avant d'accéder au pouvoir. Né sans protecteurs à la Cour, il lui fallut en trouver lui-même. C'est la raison pour laquelle son ascension est particulièrement exemplaire. Lui et sa famille doivent surmonter les obstacles qui limitent à l'époque la promotion sociale. S'il faut établir un parallèle, ce n'est sûrement pas avec Richelieu ni Mazarin, mais plutôt avec le futur cardinal Dubois, qui commença sa carrière politique comme précepteur du futur Régent. Mais sans la disparition de deux dauphins, Dubois n'aurait jamais eu accès au pouvoir. 
Né le 22 juin 1653, Fleury est baptisé Hercule ${ }^{3}$. Sa famille fait partie de cette " noblesse ancienne et illustre " qui abonde dans le Languedoc ${ }^{4}$. Son statut était élevé dans la ville de Lodève, mais son influence ne dépassait pas les limites de la région. La prospérité de cet évêché était basée sur la manufacture des draps où sa famille était active depuis des générations. Lucrèce de Rosset, sa grandmère maternelle descend des premiers drapiers de Lodève, son mari appartient à une famille de marchands. Sa famille paternelle œuvrait à l'administration financière de la province. Pierre de Fleury, son oncle paternel, trésorier général des finances de Montpellier, et intendant des gabelles pour le Languedoc, était le personnage le plus puissant de la famille ${ }^{5}$. Le père de Fleury était quant à lui receveur des tailles pour le diocèse de Lodève, office qui impliquait une certaine aisance ${ }^{6}$.

Ces deux familles, unies par le mariage des parents d'Hercule, ont une stratégie d'alliances matrimoniales destinées à asseoir leur prospérité ${ }^{7}$. Hercule n'est pas le seul fils de Jean de Fleury et Lucrèce de Rosset. Son frère aîné, Gabriel, héritier de la plupart des titres de la famille, devient conseiller à la Cour des comptes, aides et finances de Montpellier. En 1674 le lien avec la Cour des comptes est renforcé par le mariage de sa sœur avec Jean Cordurier, avocat général à la même Cour. Une autre sœur épouse, en 1680, Bernadin de Rosset, cousin issu de germain et héritier de la fortune des Rosset, et c'est leur fils qui, en 1736, reçoit le titre de duc de Rosset de Fleury crée en reconnaissance des services rendus à l'État par le cardinal Fleury. Henry de Fleury, un deuxième frère plus âgé et baron de Fozières, hérite des offices tenus par son oncle, le trésorier général. Avec Hercule, la stratégie d'ascension sociale ou politique de la famille se développe dans une autre sphère que l'office : il poursuit son chemin dans l'Église, ce qui explique le changement de prénom,

3 Le certificat de baptême se trouve parmi les papiers du cardinal de Fleury, BnF, fonds Nouvelles acquisitions françaises (désormais Naf) 22404.

4 François-Alexandre Aubert de La Chenaye-Desbois et Jacques Badier, Dictionnaire de la noblesse, $3^{\text {e }}$ éd., Paris, 1866.

5 Sur l'importance de la manufacture de draps à Lodève, et la famille de Fleury, voir Ernest Martin, Histoire de la ville de Lodève depuis ses origines jusqu'à la Révolution, Montpellier, Guiraud, 1900, vol. II, p. 216-218, et Émile Appolis, Un pays languedocien au milieu du XVIII ${ }^{e}$ siècle, Le diocèse civil de Lodève, Albi, Imp. coopérative du sud-ouest, 1951, p. 317-318 et p. 481. Pour la famille de Fleury, voir aussi Maxime de Sars, Le cardinal de Fleury, apôtre de la paix, Paris, Hachette, 1942, p. 12-15; pour ses aïeux immédiats, voir A. Vitalis, "Fleury, les origines, la jeunesse ", Annales du midi, vol. XVIII, 1906, p. 40-62.

6 Le titulaire du poste devait non seulement centraliser les sommes recueillies par les agents du fisc mais aussi fournir des avances au trésor royal.

7 Voir M. de Sars, Le cardinal de Fleury, op. cit., p. 12-15. 
André-Hercule ayant une connotation moins païenne qu'Hercule, et c'est lui qui est choisi pour représenter la famille à la Cour de France.

Dans cette perspective, la famille consent un effort important, alors qu'André-Hercule n'était que le troisième fils. Mais on le considérait comme particulièrement brillant. La famille exploita donc à plein ses contacts privilégiés pour favoriser sa carrière à la Cour. L'éducation de Fleury fait l'objet d'une stratégie à long terme. Plutôt que de l'envoyer dans l'un des collèges récemment ouverts en province, son riche oncle choisit pour lui le collège de Navarre à Paris, qui est à la fois un collège d'humanités et constitue, avec la Sorbonne, l'autre lieu d'enseignement de la théologie à Paris. Il continue ensuite sa scolarité au collège d'Harcourt, à la renommée solidement établie, où il étudie la philosophie. Selon le dictionnaire de Moréri, il a rédigé en latin et en grec ses thèses dans lesquelles il expose les principaux dogmes des philosophes athéniens. Il continue son éducation à la Sorbonne ${ }^{8}$. En 1668, clerc tonsuré au collège d'Harcourt, il est élu chanoine de la cathédrale de Montpellier. Un de ses collègues chanoines fait état de ses mérites intellectuels dans une lettre :

" J'ai souvent ouï dire au père Thomassin, qui joignait un esprit pénétrant et facile au discernement le plus fin et le plus délié, que l'abbé de Fleury était un ange pour la douceur de ses mœurs et le caractère de son esprit; que, dans le temps où il faisait sa licence, il était l'objet de l'inclination de tous ses collègues, qu'il avait fait tous actes avec éclat et avec distinction, et enfin mérité éloges d'une école où l'on sait apprécier le mérite et couronner les véritables talents " ${ }^{9}$.

Il obtient sa licence en théologie en janvier 1676 et, en juillet de la même année, il est ordonné diacre puis prêtre ${ }^{10}$.

Les familles provinciales n'avaient que des possibilités d'avancement limitées en dehors de leur province. Le patronage et l'intervention active de courti-

8 Louis Moreri, Le grand dictionnaire historique, ou Le mélange curieux de l'histoire sacrée et profane, Paris, Les libraires associés, 10 vol., 1759, art. Fleury, p. 191. Les papiers du cardinal de Fleury, $\mathrm{BnF}$, Naf 22404, révèlent qu'il est déjà au collège d'Harcourt en 1668 et avait été présent à Paris en 1666.

9 Cité dans M. de Sars, Le cardinal de Fleury, op. cit., p. 20.

10 Il a pris ses quatre ordres mineurs et sous-diaconnat à Béziers en 1674, grâce aux bons offices de Pierre de Bonzi. Voir les papiers de Fleury, loc. cit., les certificats de 1668 et 1674, et Louis de Rouvroy, duc de Saint-Simon, Mémoires de Saint-Simon. Arthur-Michel de Boislisle et Léon Lecestre (dir.), 43 vol., Paris, 1879-1930, vol. IX, p. 138-45. Pour sa licence, et ses ordinations, voir ses papiers, loc. cit., certificats du 11 juillet 1676 et du 16 juillet 1676. Voir aussi A. Vitalis, " Fleury, les origines, la jeunesse ", op. cit., 1906. 
sans revêtaient donc pour elles un caractère décisif ${ }^{11}$. Les protecteurs de Fleury étaient les Castries, ${ }^{12}$ les Noailles ${ }^{13}$, et les Bonzi.

Fleury a saisi l'occasion de lier des amitiés utiles dans les meilleurs cercles. Intelligent, droit, discret et spirituel, il possédait le charme et le savoir-vivre indispensable à toute réussite à Paris et à la Cour. La " politesse mondaine " était un des fondements de l'éducation des nobles, et le savoirvivre propre à la Cour ne pouvait s'acquérir qu'en compagnie de la plus haute

11 Les études explicitement sur le patronage, le clientélisme et les réseaux qui liaient la province et la Cour ont fait long feu, et les historiens continuent à avoir du mal à conceptualiser le rôle crucial du patronage dans le système social et gouvernemental de l'Ancien Régime. Norbert Elias n'en parle pas dans La Société de cour [1932], Paris, Calmann-Lévy, 1974. Voir P. Lefebvre, "Aspects de la fidélité en France au XVII ${ }^{\mathrm{e}}$ siècle : le cas des agents du prince de Condé ", Revue historique, $\mathrm{n}^{\circ}$ 250, 1973, p. 59-106; Jean-Louis Bourgeon, Les Colbert avant Colbert. Destin d'une famille marchande, Paris, Presses universitaires de France, 1973; Robert R. Harding, Anatomy of a Power Elite: The Provincial Governors of Early Modern France, New Haven, Yale university press, 1978; Yves Durand (dir.), Hommage à Roland Mousnier. Clientèles et fidélités en Europe à l'époque moderne, Paris, Presses universitaires de France, 1981; Roland Mousnier, "Les fidélités et les clientèles en France aux XVI ${ }^{\mathrm{e}}$, XVII ${ }^{\mathrm{e}}$ et XVIII ${ }^{\mathrm{e}}$ siècles ", Histoire sociale-Social History, vol. XV, n 29, 1982, p. 35-46; Charles Giry-Deloison et Roger Mettam, Patronages et clientélismes, 1550-1750, Villeneuve-d'Ascq : Centre d'histoire de la région du Nord et de l'Europe du Nord-Ouest de l'université de Charles-de-Gaulle-Lille III/Londres : Institut français du Royaume-Uni, 1995, surtout le chapitre d'Arlette Jouanna, " Des réseaux d'amitié aux clientèles centralisées : les provinces et la cour ", p. 21-38; Sharon Kettering, Patrons, brokers and clients in seventeenth century France, New York, Oxford university press, 1986 (qui applique pour la première fois les concepts sociologiques à la question au XVII ${ }^{\mathrm{e}}$ siècle) et son " Patronage in Early Modern France ", French Historical Studies, vol. XVII, 1992, p. 839-862. Plus récemment, les réseaux en Bourgogne et en Normandie ont attiré l'attention des historiens : Katia Béguin, Les princes de Condé. Rebelles, courtisans et mécènes dans la France du grand siècle, Seyssel, Champ Vallon, 1999; Ariane Boltanski, Les ducs de Nevers et l'État royal, Genève, Droz, 2006; Ariane Boltanski et Alain Hugon (dir.), Les Noblesses normandes (XVI ${ }^{e}-X I X^{e}$ siècles), Rennes, Presses universitaires de Rennes, 2011, p. 3-110. Il n'existe rien de comparable sur les réseaux dans le Languedoc, par exemple. Pour une vue d'ensemble, voir Peter Campbell et Chantal Grell, "La Cour et l'historiographie du pouvoir ", in M. Fontoni (dir.), The Court in Europe, Rome, Bulzoni, 2012, p. 175-192.

12 Le marquis de Castries (1611-1674) "était très bien vu à la cour pour ses mérites, l'influence de sa famille et les services qu'il rendait ", Ranchon, "Vie de Fleury " [brouillon], BnF, Naf 21795, p. 5-6 (Il existe deux versions de cette biographie presque contemporaine, un brouillon Naf 21795-7, et le BnF, Naf 2076-7, "Histoire d'André-Hercule, cardinal de Fleury " par l'abbé J.B. Ranchon, la version finale. Ce dernier ne parle pas de ces années). Lui aussi bénéficia du poids de Bonzi à la cour. En 1664, il épousa sa sœur, hérita du poste de gouverneur de Montpellier et en 1668 devint l'un des trois lieutenants généraux du Languedoc. Comme Lamoignon, il était très estimé. Voir Saint-Simon, Mémoires, op. cit., vol. III, p. 325-328.

13 Le puissant et influent clan Noailles est un autre appui languedocien : voir Roger Mettam, The role of the higher aristocracy in France under Louis XIV, thèse de doctorat, université de Cambridge, 1967 : sur Noailles, $2^{\mathrm{e}}$ partie, chapitre II, et sur Noailles dans le Languedoc, $3^{\mathrm{e}}$ partie, chapitre IV; abbé Millot, Mémoires politiques et militaires pour servir à l'histoire de Louis XIV et de Louis XV, composés sur les pièces originales recueillies par Adrien-Maurice, duc de Noailles, maréchal de France, ministre d'État, 6 vol., Paris, Teron, 1778, vol. III, surtout p. 253-254 et p. 347-348. 
noblesse, la culture de salon constituant l'essence même de la bienséance ${ }^{14}$. Fleury fréquentera assidûment le salon de Guillaume de Lamoignon, l'hôtel de Bouillon ${ }^{15}$, et l'hôtel de Noailles. Ce cheminement est décrit ainsi par SaintSimon dans ses Mémoires :

"Fleury, respectueux et d'un esprit et d'une humeur qui avoit su plaire, d'une figure qui plaisoit peut-être encore plus, d'une modestie, d'une circonspection, d'une profession qui rassuroit, gagna toujours du terrain, et il eut la fortune et l'entregent d'être d'abord souffert, puis admis dans les meilleures compagnies de la Cour, et de se faire des protecteurs ou des amis illustres des personnages principaux, en hommes et en femmes, dans le ministère et dans les premières places ou dans le premier crédit ${ }^{16}$.

Son premier protecteur menait " une vie un peu licencieuse ". Bonzi, évêque de Béziers, plus tard archevêque de Narbonne et à ce titre président des États du Languedoc, puis cardinal, était à cette époque un ambassadeur prestigieux. Son espoir de devenir ministre ayant été déçu, il passait l'essentiel de son temps en Languedoc ou en visite à Rome, mais se rendait fréquemment à la Cour. Après la mort du vieux marquis de Castries, son beau-frère, en 1674, il considérait les jeunes Castries, le nouveau marquis et l'abbé, comme ses propres enfants. Fleury était dans les meilleurs termes avec eux deux. Il passait beaucoup de temps dans leur hôtel parisien, où il était reçu et même logé ${ }^{17}$. Pendant les années 1690, leur influence alla croissant : Bonzi, avec l'aide du duc du Maine qui était gouverneur en titre du Languedoc, arrangea le mariage du marquis avec une nièce de Madame de Montespan, Mademoiselle de Mortemart-Vivonne.

Le clan Noailles faisait également partie de ce réseau languedocien. Fleury eut la chance de devenir un ami intime et fidèle de la maréchale, une des femmes les plus intelligentes et les plus influentes à la Cour de Versailles. Mère de treize enfants, elle nouait à la Cour pour ceux-ci les alliances les plus

14 Maurice Magendie, La politesse mondaine et les théories de l'honnêteté, en France, au XVII e siècle, de 1600 à 1660, 2 vol., Paris, Alcan, 1925 ; N. Elias, La Société de cour [1932], op. cit. ; Carolyn C. Lougee, Le paradis des femmes: Women, Salons, and Social Stratification in Seventeenth-century France, Princeton, Princeton university press, 1976; Antoine Lilti, Le monde des salons : sociabilité et mondanité à Paris au XVIII siècle, Paris, Fayard, 2005; Emmanuel Bury, Littérature et politesse. L'invention de l'honnête homme 1580-1750, Paris, Presses universitaires de France, 1996.

15 Sur Lamoignon (1609-1677) voir Saint-Simon, op. cit., vol. XIII, p. 142-144 et notes et la description par d'Ormesson de ces lundi soirs. Voir J.B. Ranchon, "Histoire d'André-Hercule, cardinal de Fleury ", BnF, Naf 2076, p. 10-14 et idem, brouillon, BnF, Naf 21795, p. 14-15, et Saint-Simon, Mémoires, vol. VI, p. 49-50 et appendice 3. 
avantageuses et les plus prestigieuses ${ }^{18}$. En 1698 la cote de sa famille était au plus haut : Louis XIV favorise le mariage de son fils aîné avec la nièce et seule héritière de Madame de Maintenon, Mademoiselle d'Aubigné. Une telle alliance atteignait presque le statut d'un mariage avec un prince de sang. Le duc de Noailles était gouverneur du Roussillon, et lieutenant gouverneur du Languedoc qu'il dirigeait depuis 1682. Son frère, confident de Madame de Maintenon, était depuis 1695 archevêque de Paris. À l'évidence, la famille avait la faveur particulière du roi. Dans une lettre de 1694, ce dernier lui écrivit "Croyez qu'on ne peut avoir plus d'amitié que j'en ai pour vous " ${ }^{19}$.

Si sa carrière avait bien commencé, le jeune Fleury demeurait néanmoins dans l'ombre, n'ayant ni charge à la Cour ni évêché. Selon toute évidence, Bonzi fut son principal protecteur, en particulier dans les années 1670. En 1675, grâce à lui, Fleury reçut l'autorisation d'acheter une des huit charges d'aumônier de la reine ${ }^{20}$. Son oncle la finança pour lui, à un prix supérieur à sa valeur (77 000 livres) ${ }^{21}$. Cet office lui permit de se faire de nouveaux et importants amis à la Cour ${ }^{22}$. Parmi eux, le cardinal de Bouillon était le plus prestigieux. Proche du roi grâce à ses fonctions et de son crédit personnel, il dirigeait, en tant que grand aumônier de France, la chapelle royale et ordonnançait les cérémonies religieuses les plus importantes. Durant la décennie 1670-1680, la maison de Bouillon est à l'apogée et l'hôtel de Bouillon est le centre d'un milieu où la haute noblesse côtoie quelques-uns des plus grands talents littéraires de l'époque. Madame de Sévigné, Madame de La Fayette, Corneille et Molière s'y retrouvent souvent, en compagnie de nombreux invités de moindre

18 Sur le pouvoir et l'essor du clan Noailles, sous la direction de la maréchale, voir Eveline G. Cruickshanks, Factions of the court of Louis XV, thèse de doctorat, université de Londres, 1956.

19 Abbé Millot, Mémoires politiques et militaires... de Noailles, 6 vol., Lausanne, 1778, vol. III, p. 253254 et p. 347-348.

20 "Fleury étoit connu et fort aimé de M. le cardinal de Bonzi, grand aumônier de la Reine. M. le cardinal de Bonzi lui procura un canonicat à Montpellier ; peu de temps après il le fit venir à la cour, où il lui fit acheter une charge d'aumônier de la Reine, qui se vendoit alors. ", Saint-Simon, op. cit., vol. VI, p. 49.

21 Saint-Simon explique que Jean de Fleury " s'étoit fourré parmi les valets du cardinal de Bonzi, dont il avoit obtenu la protection du temps de sa faveur à la cour et qu'il pouvoit tout en Languedoc ". Il présenta son fils " qui plut tellement à cette éminence italienne qu'il en prit soin ", voir Saint-Simon, op. cit., vol. VI, p. 46-47.

22 Chaque trimestre, deux aumôniers étaient de service et celui de Fleury commença en juillet (N. Besongne, État de la France, 2 vol., Paris, A. Besongne, 1677). C'était une charge habituellement occupée par les familles les plus en cour, et des courtisans arrogants froncèrent les sourcils quand l'oncle de Fleury l'acheta pour lui, le fils d'un percepteur d'impôt de province. Saint-Simon, Mémoires, op. cit., vol. VI, p. 49. Cela peut expliquer son prix très élevé, quelques 77000 livres quand le prix normal était de l'ordre de 50000 à 60000 livres. Ranchon, brouillon, 21795, p. 12-13. 
renommée, tel Fleury ${ }^{23}$. Évidemment, au cours de ces années 1680 et 1690 qui correspondent à l'affirmation de la gestion politique personnelle de Louis XIV, on n'y parle pas politique. Pour citer Saint-Simon, " Un ministre du temps de Louis XIV, [Torcy?] et qui avoit avec grande raison la confiance de ce Prince, m'a dit qu'il avoit beaucoup vécu avec M. de Fleury, mais qu'il ne lui avoit jamais parlé d'affaires sérieuses ${ }^{24}$.

Fleury fréquente aussi d'autres salons à cette période. Il est reçu par Pomponne et Torcy, par le maréchal de Villeroy et sa femme, et surtout par les Noailles ${ }^{25}$. L'amitié de la maréchale de Villeroy ne pouvait que profiter à Fleury. "La maréchale, devenue dévote, sembloit avoir les secrets de l'Église comme ceux du Conseil, et ne pas plus douter de son salut que de son crédit ${ }^{26}$. La conversation de Fleury était alors probablement plus littéraire que politique. Fleury côtoyait également la périphérie du cercle du duc de Bourgogne; il était " en grande liaison et commerce de lettres " avec Valincourt, secrétaire du comte de Toulouse, qui reprit plus tard la place de son ami Racine à l'Académie et celle d'historiographe royal. Fléchier, l'évêque de Nîmes, à l'époque tenu pour un écrivain de talent et considéré comme le rival de Bossuet était un ami commun de Fleury et Valincourt. Il en était assez proche pour qu'il serve d'intermédiaire par écrit entre Bossuet et le cardinal de Bouillon. L'intégration du jeune clerc provincial dans les cercles qui comptent à Paris et à la Cour a donc été exceptionnellement rapide et réussie.

Obtenue en 1675 par Fleury, la charge d'aumônier était généralement considérée comme assurant l'entrée dans l'épiscopat comme la duchesse de Brancas nous le raconte dans ses mémoires : " La chapelle du roi était, pour les jeunes ecclésiastiques, une autre espèce de séminaire qui les préparoit à entrer dans le monde, comme les mousquetaires étoient une seconde éducation pour ceux qui se destinoient au service " ${ }^{27}$. Les charges étaient considérées comme étant "le chemin ouvert aux bénéfices et aux prélatures "28. En 1680, la reine sollicite sa députation à l'Assemblée du clergé, expérience inestimable pour un futur

23 Félix Reyssié, Le cardinal de Bouillon, Paris, Hachette, 1892, p. 44-58.

24 Saint-Simon, op. cit., vol. VI, appendice 3, p. 518.

Voir J.-B. Ranchon, "Histoire... de Fleury ", BnF, Naf 2076, p. 10-14 et Ranchon, brouillon, BnF, Naf 21795, p. 14-15, et Saint-Simon, op. cit., vol. VI, p. 49-50 et vol. VI, appendice 3.

Duchesse de Brancas, Mémoires de la duchesse de Brancas, suivies de la correspondance de $M^{\text {me }}$ de Châteauroux, Eugène Asse (éd.), Paris, Librairie des bibliophiles, 1890, p. 22.

Ibid. 
évêque ${ }^{29}$. Il participe également à l'Assemblée de 1682. Dès lors, Fleury aurait dû entamer une belle carrière d'évêque. Mais les atermoiements de Louis XIV lui font perdre une bonne quinzaine d'années avant qu'il n'obtienne un évêché. Nous disposons de peu de détails sur sa vie durant les années 1680. Cependant, on le suppose à Montpellier où il a séjourné pour prêcher dans le Languedoc. Il y fait une forte impression sur Bâville de Lamoignon, l'intendant, qui devient un de ses grands admirateurs et intervient en sa faveur à la Cour. Bâville, intendant depuis 1685, travaille en étroite intelligence avec Noailles au gouvernement de la province. Fleury sera son ami jusqu'à la fin de sa vie. C'est peut-être grâce à l'influence de l'intendant, qu'il est rappelé à la Cour.

En 1690, Fleury accompagne le cardinal de Forbin-Janson à Rome qui y reçoit la calotte des mains du nouveau pape, Alexandre VIII. Une telle visite, semble-t-il, était quasiment indispensable pour un futur évêque. Le marquis de Lassay souligne son importance à Madame de La Fayette, à propos du voyage de son fils en Italie : "Presque tous les gens de sa qualité et de sa profession prennent ce tems pour voir l'Italie; c'est un voyage qui leur est quasi nécessaire dans la suite de leur vie; car il se trouve mille occasions où il est question de la Cour de Rome qui leur est fort utile d'avoir connu " ${ }^{30}$. Forbin-Janson était souvent chez Croissy, et pour améliorer une position déjà très favorable à la Cour, vu l'importance des jésuites à Versailles, il s'était déclaré ennemi juré des jansénistes.

Dès son retour de Rome, Fleury reçoit son premier bénéfice, l'abbaye de La Rivoure, qui rapporte 8000 livres par an. Cependant, c'est seulement en 1699, à l'âge de 47 ans, qu'il obtient un évêché. Dangeau écrit : "soit que le Roi le trouvât trop répandu dans le monde ou par quelque mauvais office secret, il ne donna presque point de bénéfices et ne pouvoit se résoudre à le faire évêque ${ }^{31}$. L'expérience de Fleury montre combien la promotion aux plus hautes places dépend directement de Louis XIV. Au moment où Dubois était précepteur du duc de Chartres, et donc en position d'accumuler de riches abbayes sans

29 La reine Marie-Thérèse écrivit à plusieurs évêques pour solliciter sa députation, voir A.N., O/1/3714, fol. 89. Il y fit montre d'une compétence administrative, et par conséquent fut redemandé pour l'assemblée de 1682. Plus tard il assistera aux assemblées de 1700 et 1705 . Voir sur les épisodes de 1680 et 1682, les recherches détaillées de A. Vitalis, "Fleury, les origines, la jeunesse ", art. cit., p. 27-29.

30 Marquis de Lassay, Recueil de différentes choses, 4 vol., Lausanne, M. M. Bousquet, 1756, vol. I, p. 301.

31 Philippe de Courcillon, marquis de Dangeau, Journal, E. Soulié (éd.), 19 vol., Paris, Firmin-Didot, 1854-1860, vol. VI, p. 452-3. 
même être prêtre, Fleury occupait encore une place de second rang. Il n'était cependant pas rare que le souverain tarde à récompenser un sujet méritant, simplement pour montrer qu'il était seul à décider en dernière instance.

Le succès final de Fleury est dû aux efforts déterminés et concomitants de femmes influentes et dévotes. Madame de Caylus, nièce de Madame de Maintenon, et la maréchale de Villeroy, ont parlé en faveur de Fleury à Madame de Maintenon; Madame de Noailles a fait pression auprès de son beau-frère Noailles, archevêque de Paris, pour qu'il entreprenne à nouveau le roi. Louis XIV cède finalement et lui accorde l'évêché de Fréjus, en Provence ${ }^{32}$. La manière dont cette nomination s'est faite nous révèle la justesse de l'analyse de Roland Mousnier concernant la nécessité absolue du patronage des gens de Cour pour passer le seuil devant lequel les quêteurs de charges piétinent ${ }^{33}$. Et, aussi, l'importance des femmes et d'abord de Madame de Maintenon.

L'évêque Fleury affiche le catholicisme de la Contre-Réforme et prend position contre le quiétisme. Un dossier intitulé " Touchant l'affaire de M. l'ancien évêque de Fréjus " contient la première lettre pastorale de l'évêque Fleury ${ }^{34}$. C'est un témoignage de son orthodoxie religieuse, où il presse le clergé de son diocèse de promulguer et de propager la bulle papale lancée sous la pression de Louis XIV pour condamner les Explications de maximes des saints de Fénelon.

Sous l'Ancien Régime, on le sait, les évêques remplissaient des fonctions administratives au service de la monarchie, ils calmaient les troubles dans une ville ou une province, ou bien présidaient les États (comme l'évêque d'Aix pour la Provence ou l'évêque de Narbonne en Languedoc), ou encore - responsabilités plus modestes - renseignaient le gouvernement central. Pour Fleury, il nous reste peu de documents, à l'exception des années 1707-1708. En 1713, on a les preuves d'une tentative du duc de Villars, gouverneur de la province,

32 Une lettre de Madame de Maintenon à l'archevêque de Noailles révèle que cette promotion fut vraiment due à la considération qu'avait Madame de Maintenon pour Noailles. " Je suis bien contente de la promotion, parce qu'il me semble que vous l'approuverés, Monseigneur, et qu'elle marque la considération que le Roy a pour vous. $\mathrm{M}^{\mathrm{r}}$ l'abbé de Fleuri n'estoit pas, par luy seul, un personnage a estre systot Evesque ", Madame de Maintenon à Noailles, le 22 novembre 1699, Correspondance générale, Théophile Sébastien La Vallée, (éd.), 4 vol., Paris, 1865-66, vol. IV.

33 R. Mousnier, "La mobilité sociale au XVII" siècle ", numéro spécial de XVII siècle, " Conclusion ", $\mathrm{n}^{\circ} 122,1979$, p. 75 : "À chaque stade de l'ascension, la faveur est indispensable, faveur d'un grand seigneur, d'un grand officier, puis d'un membre des cercles gouvernementaux, Chancelier, Surintendant, Prince du Sang, autre Prince, Ministre. La faveur est indispensable pour franchir les goulots d'étranglement jusqu'aux plus hauts rangs de la société, aussi nécessaire que la multiplicité des occupations".

34 Archives des Affaires étrangères (désormais AAE), Mémoires et documents, France, 1730, fol. 188198; mandement, fol. 231-234. 
de le faire nommer procureur adjoint pour l'Assemblée des communautés de Provence $^{35}$. Nous savons qu'en 1707, lors de l'invasion de la Provence, il a démontré sa loyauté envers la monarchie. Il n'y a aucune raison de croire les récits de Voltaire et Saint-Simon sur son séjour à Fréjus. L'antipathie de SaintSimon vis-à-vis de Fleury dans les années 1740, au moment de la rédaction de ses mémoires, est démontrée par sa relation erronée de l'attitude de l'évêque pendant la Guerre de Succession d'Espagne ${ }^{36}$. La correspondance de Fleury avec le secrétaire d'État nous montre en effet sa fidélité à la France et son souci de protéger son diocèse. Il reçoit Victor-Amédée de Savoie, mais renseigne sur l'armée étrangère et rassemble des fonds à Aix et à Marseille pour sa ville occupée $^{37}$. Une lettre de Chamillart à Fleury montre qu'il était très bien vu par Louis XIV : " Il me paraît qu'il [Louis XIV] est bien content de tout ce que vous me mandez, et de la conduite que vous avez tenue ${ }^{38}$. Pendant cette période il participe à l'assemblée du clergé de 1705, époque où il " eut l'honneur de faire souvent sa cour à Madame de Maintenon [...] elle connoissoit l'évêque de Fréjus de sa réputation et voulu ( $\mathrm{sic}$ ) le voir très souvent " ${ }^{39}$. Le 22 septembre 1707 , il écrit à son ami l'intendant Basville, " J'avois demandé une queste pour mes religieuses. Madame de Maintenon me l'a envoyée avec une lettre fort gracieuse. Vous voies que me voilà en faveur " ${ }^{40}$.

\section{II - Comment Fleury est-il devenu précepteur?}

La nomination de Fleury comme précepteur nous invite à réfléchir sur les qualités requises pour l'emploi. L'on peut tenter de répondre à cette question de quatre façons différentes. Tout d'abord, nous pouvons la comparer à celle d'autres précepteurs, surtout Bossuet, Fénelon et peut-être l'abbé Dubois et

35 Villars était gouverneur de la province et il a écrit à l'intendant Le Bret qu'il croyait que c'était " de l'intérêt de la Provence qu'un aussy habile et ausy honneste homme que M. de Fréjus ait part a la direction de ses affaires. ", Villars à Le Bret, le 15 août 1713, BnF, Mss fr., 8959, fol. 13-14. Fleury apparemment refusa le poste qui l'aurait associé au comité des onze qui traitait des affaires provinciales pendant les intersessions de l'assemblée.

36 Saint-Simon, op. cit., vol. XXVI, p. 85-86 : "Le Roi en fut dans une telle colère, que Torcy, ami intime du prélat, eut toutes les peines imaginables à le détourner d'éclater ". C'est de la pure invention.

37 Saint-Simon, op. cit., vol. XXVI, p. 85-86; et C. Joret, " Basville et l'épiscopat de Languedoc ", Annales du Midi, vol. VII, 1895, p. 27-29.

38 Correspondance publiée dans Saint-Simon, op. cit., vol. V, appendice V, citation p. 532.

39 J.B. Ranchon, "Histoire du cardinal de Fleury ", BnF, Naf 2076, p. 68.

40 Fleury à Basville, le 22 septembre 1707, dans C. Joret, "Basville et l'épiscopat de Languedoc ", art. cit., p. 5-50, p. 29. 
nous interroger sur le fait qu'il manquait à Fleury les qualités littéraires et érudites évidentes des deux premiers : il est loin d'être un savant connu, ou d'avoir de nombreuses publications à son actif. Une seconde approche serait de réfléchir dans l'absolu sur les qualités et connaissances nécessaires pour délivrer une bonne éducation à un futur roi. On pourrait ensuite examiner les capacités de Fleury, et chercher à savoir s'il s'est bien acquitté de ses fonctions. En réalité, il faut surtout s’interroger sur les qualités jugées nécessaires par Louis XIV et Madame de Maintenon, car ce sont eux qui ont fait le choix. La réponse est à surtout trouver dans le climat " politique " de la Cour entre 1713 et 1715, qui fait que tout dépend, pour le Roi et Madame de Maintenon, de la religion et des factions religieuses.

Il leur fallait tout d'abord un candidat qui réunisse au mieux les qualités attendues. À l'évidence, Fleury avait la grâce, le savoir-vivre et l'honnêteté d'un homme de Cour. Ce que nous savons de la première partie de sa carrière le prouve. Il était aussi très bien éduqué et s'intéressait aux questions de dogme, comme à celles de littérature et de philosophie. Quelques indications évoquent une nomination éventuelle comme précepteur des ducs de Bretagne et d'Anjou, par Marie-Adélaïde de Savoie (dont le père occupa Fréjus en 1707) ${ }^{41}$. Si tel est le cas, ceci éclaire différemment la bataille pour le préceptorat en 1715, car Fleury aurait déjà été candidat, même si les sources n’en parlent guère. Il est vrai qu'il ne figurait pas sur la liste de Fénelon comme candidat potentiel, en 1712. Mais cette liste ne peut pas être considérée comme impartiale, et Fénelon n'était plus du tout en faveur auprès de Louis XIV ${ }^{42}$.

En fait, ne pas figurer sur la liste aurait même été un atout selon Madame de Maintenon, qui voulait prendre ses distances avec Fénelon et le Quiétisme ${ }^{43}$. On a signalé plus haut que la première publication de Fleury comme évêque

41 J.B. Ranchon, "Histoire du cardinal de Fleury ", op. cit., BnF, Naf 2076, p. 106-107; voir aussi Pascale Mormiche, Devenir Prince. L'école du pouvoir en France, Paris, CNRS Éd., 2009, p. 71-72.

42 François de Salignac de La Mothe Fénelon, Mémoires sur les précautions et les mesures à prendre âpres la mort du duc de Bourgogne. Quatrième mémoire. Éducation du jeune prince, 15 mars 1712 in Louis-Aimé Martin (éd.), OEuvres de Fénelon, 3 vol., Paris, 1835, vol. III, p. 457.

43 Mark Bryant, " Partner, Matriarch, and Minister: Madame de Maintenon of France, Clandestine Consort, 1680-1715 ", dans Clarissa Campbell-Orr (dir.), Queenship in Europe, 1660-1815: The rôle of the Consort, Cambridge, 2004, p. 77-106 et Mark Bryant, Sharing The Burdens of Monarchy, Louis XIV and Madame de Maintenon, 1669-1715, "Royal Historical Society Studies " in History, Londres, à paraître, 2012. 
en 1700 fut un mandement condamnant le livre quiétiste de Fénelon ${ }^{44}$. À la différence de Pascale Mormiche dans son par ailleurs excellent article sur Fleury ${ }^{45}$, on estime donc que Fleury a clairement pris parti dans les deux grandes affaires religieuses du temps : le quiétisme et le jansénisme. Faute de pouvoir saisir ce que le roi agonisant avait en tête, tout ce que l'historien peut faire est de construire un scénario plausible conduisant à la nomination de Fleury dans le contexte de la Cour en 1715.

Sans aucun doute, pour le roi et Madame de Maintenon, la question religieuse vient au premier plan. L'attitude de Fleury envers le jansénisme est le fil conducteur de sa carrière à partir de 1711 et jusqu'à la fin de sa vie, en 1743 . Pour l'époque 1711-1715, nous avons une poignée de lettres de Fleury au cardinal de Noailles, apparemment oubliées par ses historiens, qui nous révèlent sa position sur le jansénisme.

Lorsque le cardinal de Noailles, se soumettant pour une fois à la volonté de Louis XIV, publie un mandement contre les Réflexions morales de Quesnel, livre qui est alors le symbole même du jansénisme, l'évêque de Fréjus le félicite. Sa lettre témoigne de son approbation pour la bulle Unigenitus qui condamne les 101 propositions du livre de Quesnel ${ }^{46}$. En 1713, Fleury est l'un des premiers évêques à publier la bulle dans son diocèse. Peu après, le 6 mai 1714, il publie une critique sévère des Réflexions morales, où il se révèle en parfait accord avec l'Assemblée du clergé et condamne ce qu'il regarde comme le poison du jansénisme $^{47}$. Son vigoureux plaidoyer en faveur d'Unigenitus attira l'attention du public, le mit en contact avec le cardinal de Rohan à Versailles, et fut sans doute connu de Louis XIV ${ }^{48}$.

La lettre qui suit fut écrite pour dire son accord avec une publication du cardinal de Noailles à propos des nonnes de Port-Royal. Il se range fermement

44 Lettre citée. Sur les mandements et activités de Fleury comme évêque, voir Hippolyte Espitalier, Les évêques de Fréjus du XIII e siècle à la fin du XVIII e siècle, 2 vol., Draguignan, Latil, 1898, vol. II, p. 315-372.

45 Pascale Mormiche, " Le cardinal de Fleury et l'éducation de Louis XV d'après "L'Abrégé de l'histoire de France, rois de la première et de la deuxième race” " in Chantal Grell, Werner Paravicini et Jürgen Voss (dir.), Les Princes et l'histoire au XVII et XVIII siècle, Bonn, Bouvier, 1998, p. 573-589.

46 Fleury à Noailles, le 17 octobre 1713, BnF, Mss fr., 23218, fol. 167.

47 Voir H. Espitalier, Les évêques de Fréjus, op. cit., vol. II, p. 354-366, où il cite ce mandement en longueur.

48 Voir Fleury à Rohan, Montpellier, le 26 juillet 1714, BnF, Mss fr., 17748, citée par Valentin Durand, Le jansénisme au XVIII ${ }^{e}$ siècle et Joachim Colbert, évêque de Montpellier, Toulouse, Privat, 1907, p. 26. 
dans le camp anti-janséniste et souligne la dangerosité des Réflexions morales de Quesnel.

"À Fréjus, ce 17 mai 1711

J'ai reçu, Monseigneur, il y a quelques jours les lettres dont V.E. m'a honoré du 23 du mois passé avec celle aux filles de Port-Royal. On ne peut, sans flatterie, rien de mieux écrire, de plus sage, de plus sensé et de plus touchant. Il n'y a pas de janséniste si entêté qui ne dût s'y rendre et je pense qu'elle aura tout son effet auprès de ces religieuses, comme elle a déjà eu pour deux d'entre elles. Si on ne cherchoit pas la vérité, et qu'on voulut s'entendre sans passions, on conviendroit aisément bientôt, mais comme dit fort V.E., on ne convertit point les cabales de Coeur. J'ai parlé à quelques-uns à Aix au sujet du sieur Léger : je les ai convaincus de ses torts et de ses erreurs; ils font les étonnés, ne disent pas mot et demeurent dans leurs persuasions. Ils ne font plus aucune difficulté de signer le formulaire depuis deux écrits détestables que quelqu'un du parti a fait pour éluder cette signature et le serment qui l'accompagne. Ils s'appuient fort aussi sur le P. Quesnel, et je voudrais, Monseigneur, que V.E. put avoir le temps d'examiner cet ouvrage par Elle-même, car j'ai achevé de le lire et il me paroit qu'il y a des propositions bien condamnables; je n'ai, Dieu merci, que trois ou quatre disciples du sieur Léger et suspects du jansénisme ${ }^{49}$.

Si Fleury fut anti-janséniste, il n'était pas pour autant inféodé aux jésuites.

Le passage suivant exprime très clairement ses vues à l'époque.

" Je puis vous assurer avec la plus exacte sincérité que je me suis éloigné de toute prévention en lisant le P. Quesnel, et que je ne me suis pas moins en garde contre les sentiments molinistes que contre les jansénistes. Je suis très persuadé qu'il n'y a que les décisions de l'Église qui doivent nous fixer en tout, et que nous devrons proscrire tout ce qui y est contraire. Je puis encore vous assurer que je ne me suis attaché à examiner ce livre que par l'entêtement opiniâtre que j'ai connu des gens du parti pour le défendre le regardant comme à couvert des censures à cause du nom de V.E. J'ai reconnu en eux tant de mauvaise foi, d'orgueil et de principes de révolte, que j'en ai été indigné, et le sieur Léger est celui qui m'en a le plus convaincu, aussi bien que quelques-uns de ses sectateurs qui m'ont voulu parler en sa faveur. Je conviens que l'on ne prend pas les voies ordinaires contre les abus et que la passion ou l'intérêt cherchent à envelopper dans le nombre des jansénistes des génies qui en sont fort éloignés, mais sans faire attention à ces excès et sans y prendre part on peut mettre à couvert la foi de l'Église. Elle est attaquée par bien des endroits, et cela devroit obliger ceux qui lui sont véritablement soumis à se réunir et à ne défendre que ses décisions sans entrer dans les décisions contestées. C'est le seul moyen efficace pour la soutenir, comme nous le devons, car on n'en viendra jamais à bout autrement. Mon zèle me mène trop loin et je vous en demande pardon " ${ }^{50}$.

49 Correspondance du cardinal de Noailles, BnF, Mss fr, 23217, fol. 37-38.

50 Le 11 juillet 1711, Correspondance du cardinal de Noailles, BnF, Mss fr, 23217, fol. 34-5. Voir aussi sa lettre du 16 décembre 1711 , loc. cit., fol. 41-3. 
Fleury, ayant le sentiment que l'Église était menacée, soutint la politique royale de répression du jansénisme durant les deux années qui suivirent. La position théologique de Fleury en 1715 est donc la suivante : un épiscopat uni est la clé de voûte de l'église gallicane, qui court le risque d'un schisme sur la question du jansénisme. La meilleure manière de la mettre à l'abri est de se rallier à la politique anti-janséniste de Louis XIV, et par conséquent de condamner les Réflexions morales et d'accepter Unigenitus. La défense de la hiérarchie ecclésiastique est à ses yeux fondamentale : les évêques doivent user de tous leurs pouvoirs pour empêcher le bas clergé de se rallier à la cause janséniste ${ }^{51}$.

Les troupes de choc de cette campagne anti-janséniste sont les jésuites, sous la direction, à Versailles, du père Le Tellier, confesseur du roi depuis 1709 . C'est lui qui est en partie responsable du durcissement royal envers les jansénistes. Alors que la querelle s'aggravait, il devenait de plus en plus important pour les courtisans de se ranger du bon côté, en particulier pour les ecclésiastiques. Saint-Simon, résume ainsi la situation :

"Le roi qui avoit hautement épousé ce parti [les jésuites], qui étoit exactement fermé à n'écouter que ceux qui y étoient les plus ardents, qui y avoit mis son autorité et sa conscience, qui étoit occupé ni entretenu d'autre chose, regardoit le parti opposé [les jansénistes donc] comme ennemis de l'Église et de l'État, comme républicains, comme ennemis de son autorité et de sa personne " ${ }^{2}$.

Il est clair que Fleury a choisi le parti du roi dans les trois grandes controverses religieuses de cette fin de règne. Comme nous l'avons vu, le point décisif de toute son orthodoxie religieuse fut sa position sur le jansénisme et ses efforts pour convaincre le cardinal de Noailles d'atténuer son opposition à la politique religieuse de Louis XIV.

Si l'engagement politique et doctrinal de Fleury est ainsi bien établi, nous n’avons pas de témoignage clair et fiable sur les démarches entreprises par celui-ci pour devenir précepteur. Nous ne savons même pas s'il a vraiment cherché le poste. Mais pour réussir son dessein, il lui aura fallu, comme toujours dans une société de Cour, mobiliser ses amis et protecteurs. Pendant

51 On note que sa bibliothèque personnelle dans sa maison d'Issy comprend, pour les livres publiés avant 1720, une grande majorité d'œuvres d'exégèse religieuse. Catalogue de livres du cardinal de Fleury qui sont dans son appartement à Issy, BnF, Naf 1727.

52 Saint-Simon, op. cit., vol. XXIII, p. 403 : Le 11 juin 1711, la princesse Palatine, mère du futur régent, écrit : "Il y a une guerre permanente entre les Jésuites et les jansénistes, mais les premiers ont l'appui du Roi, ce qui les renforce beaucoup. Ils tourmentent leurs ennemis de toutes les manières possibles; et ici on ne peut pas faire meilleur choix que de prendre parti contre eux ". 
ses séjours en Provence il fait plusieurs visites à la Cour, y garde ses amis et s'en fait de nouveaux.

En 1713, à soixante ans, il avait demandé la permission de quitter l'épiscopat pour raisons de santé. Pas de réponse; en décembre 1714 il avait renouvelé sa requête ${ }^{53}$. En janvier 1715, l'abbé de Castellane avait pris sa place à Fréjus, et Fleury recevait l'abbaye de Castellane, près de Reims. Fleury était ainsi libre de revenir à la Cour. L'abbaye rapportait à peine 4000 livres, mais c'était une solution provisoire, jusqu'à ce qu'on lui trouve quelque chose de mieux. Il quitte Fréjus en avril et arrive à Paris le dix-neuf de ce mois. Il restera à la Cour pendant quatre mois, avant de devenir précepteur de Louis XV par un codicille rajouté au testament de Louis XIV en août.

Comme tous les offices à Versailles, la charge de précepteur fait l'objet d'intrigues. D'un côté, il y a les jésuites, sous la direction du père Le Tellier, qui veulent choisir le précepteur afin d'accroître leur influence sur le futur roi. Étant donné les penchants religieux de Louis XIV à la fin de sa vie, Le Tellier devait croire à son succès. En face, on trouve un parti qui préfère un candidat plus modéré, de peur de voir le pouvoir de Le Tellier renforcé. Cette faction, qui rassemblait des dévots tels que le duc du Maine, Madame de Ventadour, Madame de Lévis et les Villeroy, était très proche de Madame de Maintenon, qui, pour sa part, s'opposait aux machinations de Le Tellier. Il est facile de voir que, pour ce parti, Fleury était le candidat idéal : il n'était pas jésuite, ne s'entendait pas avec Le Tellier mais, évêque très orthodoxe, il était suffisamment dévot pour rassurer le roi ${ }^{54}$. Dans cette cabale, se trouvent des amis de Fleury - les Villars, les Villeroy, les Dangeau, et Madame de Lévis - en mesure de témoigner de son érudition et de son aptitude pour le poste. Mais l'intervention de Madame de Maintenon auprès de son mari est cruciale. À cette époque de sa vie, Louis XIV a une confiance absolue en elle. Ses compétences en matière

53 Cette chronologie prouve que Saint-Simon a tort d'insinuer que l'évêque avait quitté son diocèse uniquement pour préparer sa tentative de devenir précepteur.

54 Le compte-rendu de Saint-Simon (op. cit., vol. XXVI, p. 86- 89) offre un aperçu fascinant et détaillé de la façon dont un courtisan se servait habituellement de son patron; néanmoins, il s'éloigne de la vérité sur un point important. Saint-Simon prête à Fleury une forte dissimulation et suggère qu'il adopta une attitude très religieuse uniquement pour favoriser sa carrière. Le mémorialiste se rangeait lui-même parmi les partisans des jansénistes et il noircit délibérément le caractère d'un homme qu'il détestait en lui attribuant une ambition illégitime. En réalité, Fleury ne s'est pas soudainement fabriqué une réputation de religieux pour servir ses desseins. Ses sentiments religieux tels qu'on peut les trouver dans sa correspondance privée avec les Noailles en 1711, ne diffèrent en rien de son comportement en public en 1713 et 1714. Voir aussi "Confesseurs du Roi " dans Prosper Faugère (dir.), Écrits inédits de Saint-Simon, 6 vol., Paris, Hachette, 1880-1893, vol. II, p. 479-480. 
d'éducation sont reconnues. N'a-t-elle pas été responsable de la nomination de Fénelon comme précepteur du duc de Bourgogne?

En 1715, trois de ses candidats seront choisis pour s'occuper de l'éducation du dauphin : le duc de Villeroy, gouverneur; la duchesse de Ventadour, gouvernante; et Fleury, précepteur. On peut ajouter que Madame de Maintenon, liée au clan Noailles depuis environ 1696, avait lancé en 1711 une campagne par l'intermédiaire de ses proches pour convaincre le cardinal de Noailles récalcitrant d'abandonner ses prises de position en faveur des Réflexions morales de Quesnel, et à partir de 1713 d'accepter Unigenitus, dans l'intérêt de la paix de l'Église ${ }^{55}$. Les lettres de Fleury à Noailles montrent clairement qu'il participait au même combat. Ce qui nous manque, c'est la preuve d'un lien direct entre Maintenon et Fleury sur ce sujet.

On ne se préoccupa probablement pas de choisir un précepteur au dauphin avant que Louis XIV ne tombât malade, puisque ce précepteur ne devait entrer en fonction qu'en 1717, quand le jeune Louis fêterait ses sept ans. Finalement la nomination intervint seulement une semaine avant la mort du roi. Le Tellier s'y opposa vigoureusement, mais en vain. Saint-Simon raconte le rajout au testament de Louis XIV qui conclut l'affaire :

" Il n’y demeura que Madame de Maintenon et le Chancelier. Tout aussitôt, et cet aussitôt fut un peu étrange, on apporta sur le lit une espèce de livre ou de petite table; le Chancelier lui présenta le codicille, à la fin duquel il écrivit quatre ou cinq lignes de sa main, et le rendit après au chancelier.

Versailles 23 aout 1715

Je nomme pour précepteur du jeune roi l'abbé Fleury, ancien évêque de Fréjus et pour son confesseur le père Le Tellier " ${ }^{56}$.

En définitive, parmi les critères de sélection qui ont conduit à la distinction de Fleury, il semble bien que sa fiabilité doctrinale ait été le plus déterminant.

\section{III - Devenir ministre}

On réexaminera dans cette partie la trajectoire empruntée par Fleury pour maintenir sa position à la Cour, puis pour la renforcer jusqu'à devenir, en 1720, membre de l'un des conseils de la Régence, ministre d'État en 1723 et, fina-

55 Voir Mark Bryant, "Partner, Matriarch and Minister...", op. cit., p. 97-98.

56 Journal du Marquis de Dangeau, MM. Soulié, Dussieux, de Chennevières, Mantz, de Montaiglon (éd.), 19 vol., Paris, 1854-1860, vol. XVI, p. 285. 
lement, en 1726, premier ministre sans le titre. Ses contemporains, souvent suivis par les historiens, ont a posteriori insisté sur l'ambition de Fleury. Aussi, l'historien doit-il écarter les écrits des adversaires de Fleury des années 1740, pour mieux reconstruire une micro-histoire de la vie politique. L'obligation de défendre sa position et de l'améliorer était ainsi commune à tous les courtisans et les ambitions de Fleury ont nécessairement évolué au fur et à mesure des opportunités qui s'ouvraient à lui, ses origines médiocres lui interdisant d'afficher d'emblée de trop grandes prétentions. Sa promotion exigeait pourtant plus que des opportunités : il lui fallait pour arriver au pouvoir en 1726 maîtriser les arcanes de la politique à la Cour et faire preuve d'un réel talent comme ministre des affaires de l'Église puis de l'État. Le passage du poste de précepteur ayant la confiance du roi enfant à la position de ministre d'État est un parcours semé d'embûches. Son ascension se fit en donc plusieurs étapes.

La première fut de survivre à l'hostilité de la Cour, et le meilleur parti était d'exceller dans sa fonction, de conserver le soutien de sa faction tout en gagnant la confiance du Régent qui avait le pouvoir de le renvoyer. En 1717, l'âge du roi lui laissait seulement cinq années pour assurer chaque jour son éducation, avant que sa majorité n'intervienne, à son treizième anniversaire. Louis serait alors en droit d'arrêter l'enseignement de Fleury et de choisir quelqu'un d'autre pour l'éduquer et le conseiller.

Il ne fait pas de doute que Fleury préparait ses enseignements avec soin, insistait sur la discipline, travaillait dur lui-même, et offrait à son élève un régime d'étude à la fois exigeant et à la page. Son Abrégé de l'histoire de France, aussi bien que les nombreux volumes de devoirs de Louis XV qui ont été conservés, confirment qu'il avait soigneusement réfléchi au contenu de ses $\operatorname{cours}^{57}$. Ce qui nous intéresse ici est que le précepteur a mis cette période à profit pour gagner l'estime du Régent, avec à la clé l'octroi de plusieurs faveurs notables. En 1717-1718 il entre tout naturellement à l'Académie française, où son action d'éclat fut d'attaquer l'abbé de Saint-Pierre pour sa dénonciation du style monarchique de Louis XIV.

En 1720, deux changements significatifs de la politique du Régent trouvèrent Fleury déjà bien placé pour faire de nouveaux progrès. Effectuant un spectaculaire retournement diplomatique, qui était dans l'intérêt de la Maison d'Orléans comme de la France, une alliance fut conclue avec l'Angleterre et

57 L’Abrégé de l'histoire de France écrit pour le jeune Louis XV par le cardinal de Fleury, précédé par "L'éducation de l'enfant-roi ", Chantal Grell (éd.), Archives départementales des Yvelines, 2004. 
l'Espagne, qui devait être scellée par le double mariage du jeune roi avec l'Infante d'Espagne et de la fille du Régent avec l'héritier du trône d'Espagne. Parallèlement, la politique d'" explications " destinée à permettre un compromis entre les évêques jansénistes français et le pape à propos d'Unigenitus, que le Vatican refusait, fut abandonnée au profit d'un durcissement vis-à-vis des jansénistes - en vue aussi de la préparation de la future alliance espagnole. Tout ceci conduisit en 1720 à la création d'un Conseil de conscience, dont Fleury fut nommé membre ${ }^{58}$. Cette nomination lui donna l'occasion de faire montre de ses capacités d'administrateur. Il serait cependant faux de dire, comme Georges Hardy : "La politique du Conseil de conscience, à partir de 1720, peut être regardée, dans son ensemble, comme l'œuvre personnelle de Fleury " ${ }^{59}$. En fait, les pouvoirs du Conseil étaient partagés entre ses membres et il était plutôt dominé par Dubois, Fleury avait un rôle influent mais relatif ${ }^{60}$.

La véritable force de Fleury venait des liens étroits qu'il est arrivé à garder avec la coterie qui l'avait soutenu, connue aujourd'hui comme la "Vieille Cour ", et de son amitié avec le Régent et ses amis, Saint-Simon en tête. Mais surtout, en 1721, Fleury était sans conteste la personne envers qui Louis XV avait l'attachement affectif le plus fort. En août de la même année, Saint-Simon écrit au cardinal Gualtieri pour lui raconter la maladie du roi qui dura cinq jours et provoqua à l'époque une grande inquiétude. Il raconte qu'après la "saignée le soulagement fut aussytost et il fut guay une heure avec M. de Fréjus qu'il voulut absolument qu'il luy promist de se coucher ". Deux jours plus tard, il allait mieux, et le duc de préciser que " On ne peut montrer plus d'esprit, de douceur et de tranquillité qu'il a fait en cette occasion, et sa prédilection unique pour M. de Fréjus n'a jamais tant paru "61.

À cet instant, sa proximité avec le jeune roi rendit Fleury indispensable au Régent. Ce dernier avait besoin que Louis XV se prononce personnellement en Conseil et approuve le mariage espagnol. Pour Fleury, les fiançailles de

58 Il est en 1717-1718 membre du Conseil pour les affaires ecclésiastiques de la Polysynodie : Ranchon, 2076, p. 202-207; A.A.E., Mém. et Doc., France, 139, fol. 194.

59 Georges Hardy, Le cardinal de Fleury et le mouvement janséniste, Paris, 1925, H. Champion, p. 11-12. Il est à noter que les jugements portés par G. Hardy sont souvent à réviser à la lumière de recherches plus récentes sur le jansénisme et la politique. Voir à ce propos Peter R. Campbell, Power and Politics in France, 1720-1745, Londres, Routledge, 1996.

60 Voir Michel Antoine, Le Conseil du Roi sous le règne de Louis XV, Genève, Droz, 1970, p. 109-113.

61 Voir Étienne Charavay, "Louis de Rouvroi duc de Saint-Simon. Maladie de Louis XV; promotion de l'abbé Dubois au cardinalat " Revue des Documents historiques, 1875, p. 145-148, p. 146. La lettre est datée juillet au lieu d'août par erreur, selon E. Charavay. 
Louis XV étaient l'occasion de démontrer l'ampleur de son emprise sur son élève. Il ne laissa pas passer l'opportunité et il ne lui fallut qu'une demi-heure laborieuse pour convaincre le jeune roi. "Sans l'évêque de Fréjus, qui encore s'etoit fait attendre, ils ne savoient ce qui en seroit arrivé. L'angoisse avoit été si forte, qu'ils [Dubois et le Régent] s'en sentoient encore tous deux "62. Dès 1720, Fleury avait ainsi établi une relation très étroite avec son élève. En 1721-1722, la dépendance royale à son égard fut probablement accrue du fait du climat d'intrigues qui entouraient Louis XV. Cette relation de confiance n'avait rien de spontanée, Louis, par exemple, détestait son gouverneur, le pompeux Villeroy.

Toutefois, la confiance de l'enfant n'aurait pas suffi en elle-même à acquérir le pouvoir, l'habileté de Fleury compta aussi. À l'automne 1721, le Régent lui offrit l'archevêché de Reims, rendu vacant par la mort de Mailly. Non seulement Fleury déclina l'offre mais s'arrangea pour qu'il aille à un Rohan-Guéméné et pas à un candidat Noailles. On a l'impression que la relation patron-client s'inversait. Fleury est invité à choisir entre sa dignité et son obligation de servir le Régent, mettant fin à son commerce quotidien avec le roi, ou de rester le précepteur et le confident du roi et de tenter sa chance. Qu'il choisisse la possibilité du pouvoir politique est révélateur de son attachement au roi, d'une ambition née des circonstances, et de son aptitude à évoluer dans le perpétuel jeu d'échecs que fut la vie de Cour.

La preuve suivante de son habileté diplomatique survint à l'été 1722 . L'arrière-fond était la montée d'un conflit entre factions au printemps, après que le Régent a montré des signes de maladie grave faisant craindre pour sa vie en janvier et mars. La Vieille Cour restait anti-Orléaniste et, en février, le duc de Bourbon quitta le Conseil de régence en signe de protestation contre l'arrivée du cardinal de Rohan, dont on savait qu'elle préparait celle de Dubois. Aux prises avec une telle opposition et avec des alliés aussi dangereux, les chances pour Dubois et le Régent de garder le pouvoir à un an seulement de la majorité du roi semblaient faibles - une situation que les historiens ont sous-estimé. Le Régent et Dubois visaient Villars, dont Louis XIV était proche, et attaquaient la Vieille Cour, avec le gouverneur royal et le puissant duc de Noailles exilés fin juin.

Que devait faire Fleury? Villeroy était son bienfaiteur, il lui devait une certaine allégeance. Sa réponse fut un coup de théâtre. Le 17 août, sans rien dire, il quitta subitement la Cour de nuit, plongeant son élève dans le désarroi. 
Naturellement, il fut rappelé sur le champ sur ordre de Louis, prouvant ainsi qu'il était indispensable : "Vous vous êtes assez reposé; j'ai besoin de vous, revenes donc au plustost "63. Il avait réussi à la fois à régler sa dette vis-à-vis de Villeroy et à survivre. Comme Barbier le souligne, "C'est un homme de beaucoup d'esprit, et ce coup est d'une grande politique. Il a mis M. le Régent hors d'état de l'exiler à présent; aussi M. le Régent en a-t-il été très chagrin " ${ }^{64}$. Mathieu Marais était mieux informé :

" M. de Fréjus n'a point été arrêté. Il est parti en chaise sans rien dire à personne on ne s'est aperçu de son départ qu'à la leçon du Roi. On a envoyé sur sa piste et il a été trouvé à Courson chez M. de Bouillé, M. Des Fors qui lui a été envoyé a rapporté une lettre que le prélat a écrite au Roi où il lui marque qu'il est parti à cause d'un mal de tête qu'il avoit et qu'il craignoit qu on ne lui donnât pas de congé. Le Roi lui a écrit de se guérir promptement de son mal de tête parce qu'il avoit affaire de lui. Cette retraite est généralement blâmée et soupçonnée de mauvais desseins. Il veut faire croire qu'il n'a point eu de part à la disgrâce du maréchal de Villeroy et cependant on sait le contraire. On croit aussi qu'il veut par malignité rendre le Régent odieux et que le public dise que le précepteur n'a pas voulu rester auprès du Roi après qu'on lui a ôté son gouverneur parce qu'il ne veut point prendre part à tout ce que l'on pourra entreprendre. Le public en effet parle ainsi et le Régent est très fâché de cette malice. S'il punit l'évêque on dira qu'il ôte au Roi tous ceux qui le pouvoient instruire et avoient sa confiance. S'il ne le punit pas on dira ou qu'il le craint ou qu'il est d'accord avec lui. Embarras de toutes parts. Mais le Régent espère que la vérité se découvrira bientôt "65.

Le Cardinal Dubois devint premier ministre le 22 août 1722 et fut informé en septembre 1722 que la cabale de Le Blanc-Belle Isle espérait convaincre le roi de changer de ministres après la majorité. Une partie de la riposte du Régent et du Cardinal fut de profiter de l'éducation de Louis XV pour l'habituer à travailler avec eux : un document, cité ici pour la première fois et donné à Clairambault par le valet du Duc d'Orléans le 19 avril 1738, relate :

" Pour former le Roy et luy donner les Instructions nécessaires pour gouverner l'État M. le Duc d'Orleans voyant approcher la majorité propose d'avoir des conversations particulières avec sa Majesté. Il fut traverse par M. le Marechal de Villeroy qui voulut en qualité de gouverneur y assister, et ce ne fut qu'âpres son éloignement qu'elles eurent lieu.

Le Duc d'Orleans ne prit point d'heures ny de jours fixes pour ce travail, il alloit chez le roy comme a son ordinaire avec le Portefeuille de signatures, et souvent

63 AAE, M.D, 139, fol. 173.

64 E.J.F. Barbier, Chronique de la Régence et du règne de Louis XV (1718-1763), ou Journal de Barbier, 8 vol., Paris, Charpentier, 1857, vol. I, p. 235.

65 Matthieu Marais, Journal et Mémoires... sur la régence et le règne de Louis XV, M. de Lescure (éd.), 4 vol., Paris, Didot, 1863-1866, mardi 18 août 1722, p. 331-332. 
il revenoit sans qu'il eut este ouvert. J'ai scu de M. le Duc d'Orleans que les jours qu'on n'ouvrait point ce portefeuille estoient les jours les mieux employés par ce que les trois heures qu'il restoit avec sa Majesté se passoient en conversation qui avoient pour objet les grands principes du gouvernement. Il a paru que M. le Duc d'Orleans se preparoit avant d'aller chez sa Majesté, je l'ay vu lire quelquefois un chapitre du Testament Politique du Cardinal de Richelieu et quelquefois d'autres mémoires "66.

Il s'en suivit inéluctablement, juste après la déclaration de majorité du roi en mars 1723, que le duc d'Orléans présida le Conseil, avec Dubois comme premier ministre. Mais un fait singulier a été négligé, même par Michel Antoine : la même lettre de Dubois dont les historiens usent pour nous dire qui appartenait au Conseil, établit sans conteste que l'évêque de Fréjus était ministre ${ }^{67}$. Pour l'ancien précepteur, être devenu membre du nouveau Conseil royal d'État et donc ministre en compagnie seulement de Philippe d'Orléans, du duc de Chartres (son héritier), du duc de Bourbon et du premier ministre Dubois était une formidable réussite. Dubois écrivit au pape, demandant pour Fleury un chapeau de cardinal. L'étude micro-historique du parcours de Fleury que nous avons menée contribue ainsi à éclairer les rapports entre l'éducation de Louis XV et la carrière de son précepteur ${ }^{68}$.

Fleury était tout à la fois le client et le " champion " d'un cercle languedocien influent à la Cour, dont la fréquentation lui permit également d'acquérir cet indispensable savoir-vivre qui fut un de ses atouts. La formidable élévation

66 BnF, Clairambault 529, p. 93. Voir aussi le rapport de l'ambassadeur hollandais, Van Hoey, qui confirme cette interprétation, dans Jules Flammermont, Correspondances des agents diplomatiques étrangers, Paris, Imprimerie nationale, 1896, p. 247-260 : "On comptait par l'exil du Chancelier du duc de Noailles et du maréchal de Villeroy avoir rempli le premier point et pour le second il se forma un plan entre MM. le duc d'Orléans, le duc de Bourbon et le premier ministre pour commencer l'instruction du Roi laquelle en accoutumant ce prince à ne traiter les affaires qu'avec ces trois personnes devait jeter les fondements de la nouvelle forme d'administration qu'on voulait établir pour la majorité. Ces instructions ont été continuées jusqu'ici avec tout le succès qu'on en attendait. M. le duc d Orléans y a même ajouté des entretiens particuliers où il voit le Roi tout seul et dans lesquels on dit que ce prince s'est beaucoup accoutumé à lui. " (p. 253).

67 Michel Antoine, Louis XV, Paris, Fayard, 1989, chapitre II; voir Dubois au marquis de Rossi, le 8 mars 1723, BnF, Mss. fr., 10681, fol. 38 : "l'ordre du Conseil ou Sa Majesté doit assister a ceste regle, elle tient le dimanche et le mercredy le Conseil d'estat compose de Sa Majesté, de M. le Duc d'Orléans, de M. le Duc de Chartres, de M. le Duc, du ministre et de M. l'evesque de Frejus, dans lequel on délibère de toutes les affaires étrangères et des grandes résolutions ".

68 Le long article en deux parties, par Gaston du Fresne de Beaucourt, "Le caractère de Louis XV ", Revue des questions historiques, t. III, 1867, p. 172-217, deuxième partie, t. IV, 1868, p. 181-254, rarement cité, reste à mes yeux la meilleure analyse du caractère de Louis XV, malgré un ton moralisant propre à l'époque. Beaucourt avait consulté pratiquement tous les documents utilisés par ses successeurs pour l'éducation de Louis XV et pour ses attitudes pendant tout son règne; voir aussi Bernard Hours, Louis XV, un portrait, Toulouse, Privat, 2009, p. 139-198. 
politique de Fleury est due à la fois à sa réussite pédagogique et à sa faction. Son positionnement religieux était d'une importance capitale pour Louis XIV et Madame de Maintenon. Fleury était le protégé et l'avocat d'un parti important à la Cour, et il avait fait ses preuves comme administrateur compétent d'un évêché. Son bon caractère lui permit de nouer une relation étroite avec son élève. Bien remplir sa fonction d'éducateur était essentiel pour durer et réussir sa carrière politique. Surtout, sa discrétion et le peu de visibilité de ce parfait homme d'Église semblent avoir été ses armes maîtresses, ainsi que son désintéressement affiché, au moins si on le compare à celui des nombreuses factions qui assiégeaient le roi.

Trois témoignages inédits, au final, tendent à confirmer cette hypothèse. Une dépêche de Jean Le Chambier, l'agent diplomatique de la Prusse, témoignait en 1721 d'une prescience peu commune :

"L'évêque de Fréjus a la réputation d'avoir beaucoup d'esprit et fort peu d'ambition, tous ceux qui désirent de se rendre maitre de la confiance et de l'esprit du Roy se flattent d'avoir cet évêque dans leurs interêts; le Marechal de Villeroy le regarde comme un homme qui lui est entièrement attaché par reconnoissance, parce qu'il a contribué plus que personne a lui procurer la place de Précepteur du Roy. Madame de Ventadour, le Cardinal de Rohan et tous ceux de sa maison croyent que l'évêque de Fréjus leur est absolument devoue. M. le Régent et M. le Cardinal Dubois se flattent aussi suivant toutes les apparences de la même chose; il peut cependant arriver qu'aucun de ces trois partis n'a raison de penser comme il fait a l'égard de cet Evêque qui affectant toujours une ambition très médiocre continuera cependant de faire dans la confiance du Roy le plus de progrès qu'il lui sera possible, afin de pouvoir un jour devenir le seul et principal Ministre de ce prince ${ }^{69}$.

En 1726, quand Fleury devint premier ministre officieux, le duc de Richelieu écrivit à Madame Duras :

"Le fardeau dont s'est charge M. de Fréjus paroitroit pesant a tous les hommes; juges ce que cela doit paroistre a quelqu'un aussy doux et paresseux que luy, et je suis très persuadé qu'il est fort fache de le porter, et qu'il cru ne pouvoir s'en dispenser, croiant etre scur qu'il n'a entre aucune ambition dans ce qu'il a fait : mais comme cet un homme qui a des amis, mais qui n'influent point dans les principes qu'il s'est fait, et dans les petits arrangemens qu'il a, je ne suis pas surpris de ce qu'il a fait au Marechal de Berwick, et beaucoup de gens qui croiront qu'il n'y a qu'a se baisser et en prendre ne soient attrapes et mecontens. Car c'est un homme qui veut le bien, et sur qui les amis ne feront pas tant qu'ils pensent, hors sur le detail des finances et de la guerre ou je crois bien qu'il les governera tout seul, et que beaucoup de gens se tromperont dans ce qu'ils

69 AAE, C.P., Prusse, 70, fol. 153. 
croient qu'il faira; car je crois le connoitre, et que tout le monde ne le connoist pas, vous verres si je ne trompe ${ }^{70}$.

Ce tableau est confirmé par les fines observations de Richer d'Aube :

"Pendant longtemps son ambition ne se portoit qu'a luy procurer le pouvoir entier dans les affaires ecclesiastiques; c'etoit asses alors pour luy qui n'etoit pas ne ambitieux, mais cela n'etoit pas suffisant pour ceux qui se croyant surs de le gouverner toujours desiroient pour leur avantage de le voir maitre de tout. Ces personnes la aidees par le mauvais succes du gouvernement de M. le Duc convainquirent enfin le Prelat 1 que M. le Duc perdoit tout, 2 qu'il n'y avoit que luy Prelat qui en faisant deplacer ce Prince, pust le remplacer avec succes; 3 qu'il en scavoit assez pour gouverner tout le Royaume, et qu'il ne pouvoit pas se dispenser de se faire nommer $1^{\text {er }}$ ministre ${ }^{71}$.

À ces quelques témoignages écrits à chaud, suivront les portraits rédigés au moment de sa mort ou peu après, qui dissimulent mal l'hostilité de leurs auteurs et une tendance marquée à minorer ou ignorer ses incontestables qualités ${ }^{72}$. En effet, son long règne avait fait beaucoup de déçus parmi ceux qui visaient des promotions et des avancements; en 1743 l'opinion publique était fatiguée du vieux cardinal et regardait avec sympathie à la prochaine arrivée de Louis XV aux affaires. Du coup, la subtilité de Fleury devint duplicité, sa patience de l'irrésolution, son sens de l'économie de l'avarice et sa prudence diplomatique du pacifisme honteux. La meilleure manière de retrouver un peu de vérité sur cet ecclésiastique habile et secret est, semble-t-il, de confronter les images colportées par les contemporains avec une micro-histoire du contexte politique dans lequel il se débattit. C'est en tout cas ce que nous avons voulu faire à propos de sa montée en puissance au temps de son préceptorat.

\author{
Peter R. CAMPBELL \\ Université de Versailles-Saint-Quentin, \\ université du Sussex \\ États, Société, Religion - EA 2449, \\ peterr.campbell@virgin.net
}

70 Richelieu à Silly, 20 Juillet 1726, Bibliothèque Victor Cousin, Fonds Richelieu, 31, fol. 91.

71 Richer d'Aube, "Réflexions sur le gouvernement de France ", BnF, Naf 9511, p. 23.

72 Voir F.J. de Pierre, cardinal de Bernis, Mémoires et lettres de François Joachim de Pierre, F. Masson (éd.), 2 vol., Paris, Plon, 1878, vol. I, p. 47-48; F.V. Toussaint, Anecdotes curieuses de la cour de France sous le règne de Louis XV, P. Fould (éd.), Paris, Plon, 1908, p. 44-46 ; René Louis de Voyer, marquis d’Argenson, Journal et mémoires, E.J. Rathéry (éd.), 9 vol., Paris, 1859-1867, vol. I, p. 212 214. 